\title{
Sodium Acetate Responses in Saccharomyces cerevisiae and the Ubiquitin Ligase Rsp5
}

\author{
Akaraphol Watcharawipas, Daisuke Watanabe and Hiroshi Takagi* \\ Division of Biological Science, Graduate School of Science and Technology, Nara Institute of Science and Technology, Nara, \\ Japan
}

Recent studies have revealed the feasibility of sodium acetate as a potentially novel inhibitor/stressor relevant to the fermentation from neutralized lignocellulosic hydrolysates. This mini-review focuses on the toxicity of sodium acetate, which is composed of both sodium and acetate ions, and on the involved cellular responses that it elicits, particularly via the high-osmolarity glycerol (HOG) pathway, the Rim101 pathway, the P-type ATPase sodium pumps Ena1/2/5, and the ubiquitin ligase Rsp5 with its adaptors. Increased understanding of cellular responses to sodium acetate would improve our understanding of how cells respond not only to different stimuli but also to composite stresses induced by multiple components (e.g., sodium and

OPEN ACCESS

Edited by:

John P. Morrissey,

University College Cork, Ireland

Reviewed by:

Paola Branduardi,

Università degli studi di Milano

Bicocca, Italy

Gianluca Bleve,

Istituto di Scienze delle Produzioni

Alimentari (ISPA), Italy

*Correspondence:

Hiroshi Takagi

hiro@bs.naist.jp

Specialty section:

This article was submitted to Microbial Physiology and Metabolism,

a section of the journal

Frontiers in Microbiology

Received: 21 July 2018

Accepted: 28 September 2018

Published: 16 October 2018

Citation:

Watcharawipas A, Watanabe $D$ and Takagi H (2018) Sodium Acetate Responses in Saccharomyces cerevisiae and the Ubiquitin Ligase Rsp5. Front. Microbiol. 9:2495. doi: 10.3389/fmicb.2018.02495 acetate) simultaneously. Moreover, unraveling the characteristics of specific stresses under industrially related conditions and the cellular responses evoked by these stresses would be a key factor in the industrial yeast strain engineering toward the increased productivity of not only bioethanol but also advanced biofuels and valuable chemicals that will be in demand in the coming era of bio-based industry.

Keywords: Saccharomyces cerevisiae, ubiquitin ligase, Rsp5, sodium acetate responses, P-type ATPase sodium pumps, Ena1/2/5

\section{INTRODUCTION}

The budding yeast Saccharomyces cerevisiae is an important microorganism for the production of alcoholic beverages, bread, and bioethanol, as well as other biochemicals due to its wellknown ability during the fermentation process. S. cerevisiae cells possess relatively high ethanol productivity, and strong gassing power required for making dough, as well as produce distinct flavor for alcoholic beverages and bakery products (Shima and Takagi, 2009; Sasano et al., 2012a; Shiroma et al., 2014; Arshad et al., 2017). They also have lower nutrient requirement for growth and higher acid tolerance than lactic acid bacteria, which make them potentially useful for lactic acid production (Sugiyama et al., 2014). In the last decades, there has been increased interest in using $S$. cerevisiae for the production of other high value-added chemicals, e.g., isobutanol, branch-chain alcohols, amino acids, $\beta$-glucan, and lactic acids (Baek et al., 2017; Generoso et al., 2017; Mongkontanawat et al., 2018; Takpho et al., 2018). To meet these demands, researchers have considered the feasibility of using yeast cells in the presence of numerous stress conditions, e.g., weak acids, freeze-thaw, high sugar contents, oxidative treatment, and high temperature (Nakagawa et al., 2013; Sugiyama et al., 2014; Kitichantaropas et al., 2016), as well as several growth and/or fermentation inhibitors derived from feedstock biomass (Sasano et al., 2012b; Ishida et al., 2017; Jayakody et al., 2018). Thus, understanding the cellular responses of yeast in adaptation to these harsh conditions will be a key to improving yeast strains for future industrial applications. 
Second-generation production of fuels and chemicals e.g., bioethanol involves the utilization of lignocellulosic biomasses such as rice straw, wheat straw, bagasse, corn fiber, and corn stover as a feedstock. These materials are comprised of $40-50 \%$ cellulose, 20-30\% hemicellulose, and 10-25\% lignin (Anwar et al., 2014). To release sugars (monosaccharides/disaccharides) from these biomasses, several hydrolytic processes with acid/base or enzyme are employed (Limayem and Ricke, 2012). However, not only sugars, but also growth/fermentation inhibitors including furfural, 5-hydroxymethylfurfural, vanillin, glycolaldehyde, and acetate are generated (Iwaki et al., 2013; Jonsson and Martin, 2016; Jayakody et al., 2017). In contrast to other inhibitors that can be reduced by the optimization of hydrolytic processes, acetate released from highly acetylated hemicellulose tentatively exists in lignocellulosic hydrolylates over $10 \mathrm{~g} / \mathrm{L}$ at $\mathrm{pH}$ 56 (Palmqvist and Hahn-Hagerdal, 2000; Klinke et al., 2004; Almeida et al., 2007). Many studies have shown that acetate exerts an inhibitory effect on the growth and fermentation ability of S. cerevisiae cells (Pampulha and Loureiro-Dias, 1989; Larsson et al., 1999; Bellissimi et al., 2009). In addition, recent studies have demonstrated that acetate in the presence of sodium exerts higher growth inhibition than that in the presence of potassium (Pena et al., 2013), and sodium acetate exhibits higher cellular toxicity than sodium chloride at equal molar concentration, suggesting a synergistic inhibitory role of sodium and acetate (Watcharawipas et al., 2017). In terms of application, these findings underscore the importance of sodium acetate stress in the growth and fermentation from neutralized lignocellulosic hydrolysates.

\section{SODIUM AND ACETATE STRESSES: TOXICITY AND ADAPTIVE MECHANISMS FOR YEAST CELLS}

Acetic acid is a weak organic acid with low lipophilicity $(\mathrm{pKa}=4.75)$. It can enter yeast cells either by passive diffusion across the plasma membrane or facilitated diffusion via the aquaglyceroporin channel Fps1 (Mollapour and Piper, 2007). At the cytosolic $\mathrm{pH}$, acetic acid dissociates to acetate anions and protons in the cytoplasm, causing intracellular acidification and growth inhibition due to the perturbation of cytosolic $\mathrm{pH}$ homeostasis, which affects several cellular activities including signal transduction, metabolic functions, protein interaction, and cell division (Dechant et al., 2010; Young et al., 2010; Orij et al., 2012; Ullah et al., 2012; Stratford et al., 2013; Fernandez-Nino et al., 2015). Therefore, removing excess protons from the cells by the plasma membrane $\mathrm{H}^{+}$-ATPase Pmal and collecting protons inside the vacuole by the vacuolar proton pump V-ATPase are suggested to be necessary for normal cytosolic $\mathrm{pH}$ maintenance and cell growth recovery under acetic acid stress conditions (Martinez-Munoz and Kane, 2008; Ullah et al., 2012; Stratford et al., 2013; Smardon and Kane, 2014). Moreover, the released acetate anions also negatively affect yeast cells by increasing the internal turgor pressure that leads to cell growth inhibition (Mollapour et al., 2008). In addition, the depletion of intracellular ATP pools is postulated to occur as a result of the ATP utilization by the plasma membrane $\mathrm{H}^{+}$-ATPase Pmal and the vacuolar proton pump V-ATPase to pump protons out of the cells and into the vacuole, respectively (Ullah et al., 2013). Acetic acid also negatively affects the uptake of some nutrients, including glucose, tryptophan, histidine, lysine, leucine, uracil, and phosphate, which is possibly caused by either the reduction of intracellular ATP required for mediating the nutrient uptake or the downregulation of the involving genes such as HXT1, HXT3, $B A P 2$, and GAP1 genes (Kawahata et al., 2006; Ding et al., 2013). Moreover, programmed cell death was also triggered by high concentrations of acetic acid (Ludovico et al., 2002).

To cope with these cellular toxicities from acetic acid stress, $S$. cerevisiae utilizes the high-osmolarity glycerol (HOG) pathway to transduce acetic acid responses (Mollapour and Piper, 2006). The Hog1 mitogen-activated protein kinase (MAPK) phosphorylates Fps1, which triggers its ubiquitination, endocytosis, and degradation in the vacuole, thereby rendering yeast cells resistant to acetic acid (Mollapour and Piper, 2007). In addition to Hog1, the acetic acid-responsive transcriptional activator Haal also plays a pivotal role in acetic acid responses (Mira et al., 2011). Haal functions by regulating the transcription of various genes via the Haal-responsive element (HRE) in their promoter regions (Mira et al., 2011). These genes belong to the so-called Haal regulon, and include TPO2, TPO3, SAP30, HRK1, SPI1, and YGP1. The drug: $\mathrm{H}^{+}$antiporters Tpo2 and Tpo3 are reported to play an important role in intracellular acetate anion extrusion (Fernandes et al., 2005). It has also been suggested that the component of Rpd3L histone deacetylase complex Sap30 and the protein kinase Hrk1 are crucial for decreasing intracellular acetate contents (Mira et al., 2010). The cell wall proteins Spil and Ygp1 have been suggested to replenish the yeast cell wall to prevent the re-entry of acetic acid by direct diffusion (Fernandes et al., 2005; Simoes et al., 2006; Mira et al., 2011). In addition, it was shown that both laboratory and industrial strains of $S$. cerevisiae constitutively expressing HAA1 exhibited significantly improved cell growth and initial fermentation rates under acetic acid stress (Tanaka et al., 2012; Inaba et al., 2013). Therefore, molecular breeding of industrial yeast strains lacking FPS1 or overexpressing HAA1 could be regarded as a promising strategy for improving acetic acid tolerance in yeast cells.

On the other hand, the $\mathrm{pH}$ of lignocellulosic hydrolysates after pretreatment can be increased up to the range of 5 to 6 due to neutralization (Guo and Olsson, 2014; Wilkinson et al., 2016; Bazoti et al., 2017). Under this $\mathrm{pH}$ condition, which is higher than the $\mathrm{pKa}$ of acetic acid (4.75), acetic acid molecules are largely present as acetate anions with lower toxicity (Mollapour et al., 2009). However, the counter ions of acetic acid (e.g., sodium) play an important role in the toxicity of acetate at higher $\mathrm{pH}$ (Pena et al., 2013; Watcharawipas et al., 2017). Sodium ions inhibit the growth of yeast cells via two phenomena: (i) a high concentration of sodium causes a hyperosmotic environment that induces the loss of cytoplasmic water from yeast cells (Hohmann, 2002); (ii) a high concentration of sodium increases intracellular sodium and decreases intracellular potassium contents, interfering proper cation homeostasis in yeast cells (Arino et al., 2010). At high concentrations, sodium enters the cells mainly by displacing potassium through transporters 
that include: (i) the high-affinity potassium transport Trk1/Trk2 system (Haro and Rodriguez-Navarro, 2002); (ii) the non-specific cation transport system named NSC1 for non-specific cation channels (Gomez et al., 1996); and (iii) the sodium-dependent phosphate transport Pi-Na ${ }^{+}$symporter Pho89 (Martinez and Persson, 1998; Serrano et al., 2002). High cytoplasmic sodium levels have been shown to negatively affect the $3^{\prime}, 5^{\prime}$-bisphosphate nucleotidase gene HAL2 (Murguia et al., 1996), whereas proper intracellular potassium concentrations have been suggested to be necessary for several enzyme functions (Lapathitis and Kotyk, 1998).

The primary way that yeast cells cope with sodium stress is by maintaining intracellular contents and osmolarity. Hog1 phosphorylates the basic leucine-zipper transcriptional factor Sko1, leading to the upregulation of a subset of defensive genes that include the stress-inducible methylglyoxal reductase gene GRE2, the antioxidant peroxyredoxin gene $A H P 1$, and the sodium pump gene ENA1 (Proft and Serrano, 1999; Proft and Struhl, 2002). Ion homeostasis also involves the post-translational regulation of the sodium/proton antiporter Nhal and the potassium channel Tok1 mediated by Hog1 phosphorylation (Proft and Struhl, 2004). To maintain suitable cytoplasmic sodium levels, surplus amounts of sodium must either be extruded through the plasma membrane by active transport via the sodium/proton antiporter Nhal and the P-type ATPase sodium pumps Ena system or sequestered in the vacuole by the activity of Nhx1 and Vnx1, the two sodium/proton antiporters located in endosomal and vacuolar membranes, respectively (Nass et al., 1997; Cagnac et al., 2007). Additionally, the basal task of Hog1 is to increase the accumulation of glycerol as a compatible solute in response to hyperosmotic stress induced by high sodium concentration through: (i) the upregulation of glycerol biosynthesis genes GPD1, GPP1, and GPP2 as well as the active glycerol uptake system Stl1 (Rep et al., 2000; Ferreira et al., 2005; Petelenz-Kurdziel et al., 2013); (ii) an increase of glycolytic enzyme phosphofructo-2-kinase activity (Dihazi et al., 2004); (iii) the limiting of the aquaglyceroporin channel Fps1 activity that exports glycerol (Lee et al., 2013). Besides HOG signaling, the alkaline $\mathrm{pH}$-sensing Rim101 pathway also plays a role in intracellular sodium homeostasis. At alkaline $\mathrm{pH}$ after being $\mathrm{C}$-terminally processed, the alkaline $\mathrm{pH}$ transcription factor Rim101 enters the nucleus to control the transcription of alkaline-responsive genes such as ENA1 (Lamb et al., 2001; Serrano et al., 2002). In general, Rim101 and Hog1 act as the independent pathways to regulate the transcription of ENA1. Interestingly, a recent study showed that although Hog1, not Rim101, is predominantly required for controlling the transcription of ENA1 under sodium chloride stress conditions, Rim101 is indispensable and has a potentially novel role in the post-translational regulation of Enal trafficking to the plasma membrane (Marques et al., 2015). However, detailed molecular mechanisms need to be further elucidated. In addition, previous studies also showed that Rim101 is required for tolerance to propionic acid stress due to its involvement in the transcriptional responses of $K N H 1$, which encodes a protein that functions in the synthesis of cell wall $\beta$-1,6-glucan; $C W P 1$, which encodes a mannoprotein that links to the $\beta-1,3-$ and $\beta$-1,6-glucan in the cell wall; $B A G 7$, which encodes a Rho GTPase-activating protein that plays a role in the synthesis of $\beta$-1,3-glucan by stimulating Rho1; and YIL029c, which encodes a protein with unknown function (Mira et al., 2009). Yeast cells lacking RIM101 also exhibit impaired vacuole acidification, leading to acidic cytosolic $\mathrm{pH}$ under propionic acid stress (Mira et al., 2009). Taken together, these findings suggested that both the HOG and the Rim 101 pathways potentially participate in the cellular responses to composite stress from a salt and weak acid - in this case, sodium and acetate.

\section{THE E3 UBIQUITIN LIGASE Rsp5 AND ITS ADAPTORS}

Rsp5 (Reverses Spt ${ }^{-}$phenotype protein 5) is the sole orthologue of the human Nedd4 E3 ubiquitin ligases in S. cerevisiae, and plays important roles in regulating physiological processes in cells - including intracellular trafficking, signal transduction, and quality control of the plasma membrane and cytosolic proteins (Dunn and Hicke, 2001; Hiraishi et al., 2009; Jarmoszewicz et al., 2012; Shiga et al., 2014) - through the interaction and ubiquitination of diverse substrate proteins. Rsp5 is composed of the N-terminal calcium-dependent phospholipid membrane binding (C2) domain, three substrate-recognizing WW domains (commonly referred to two conserved tryptophan residues in the domains), and the C-terminal catalytic ubiquitin ligase (HECT) domain (Rotin and Kumar, 2009).

The essential role of Rsp5 is attributed to its activity in the regulation of the OLE1 gene expression via ubiquitinationmediated proteolytic processing of the transcriptional activators Spt23 and Mga2 that localize at the endoplasmic reticulum (ER) (Zhang et al., 1999; Hoppe et al., 2000; Shcherbik et al., 2003, 2004). Rsp5 is known to downregulate various plasma membrane transporters for nutrients and ions as well as receptors. This downregulation contributes both to protein quality control mechanisms in which the plasma membrane proteins are misfolded (e.g., in response to heat stress) and regulatory mechanisms in which the transporters are endocytosed to restrict their activity or receptors are degraded for their desensitization. For example, the ubiquitination by Rsp 5 mediates the endocytosis of the general amino acid permease Gap1 in response to the shifting from a poor nitrogen source to ammonium ions (Springael et al., 1999) and in response to environmental stresses such as ethanol, hydrogen peroxide, high temperature, and lithium chloride (Hoshikawa et al., 2003; Shiga et al., 2014). Our previous studies also suggest that Rsp5 participates in the maintenance of stress-induced abnormal proteins through degradation or repair process (Haitani et al., 2006; Haitani and Takagi, 2008; Hiraishi et al., 2009). It has been shown that Gap1 remains stable on the plasma membrane under ethanol stress in the stress-hypersensitive $r s p 5^{\mathrm{A} 401 \mathrm{E}}$ mutant (Hoshikawa et al., 2003; Shiga et al., 2014). On the other hand, constitutive inactivation and endocytosis of Gap1 was effectively mediated by the $r s p 5^{\mathrm{T} 357 \mathrm{~A} / \mathrm{K} 764 \mathrm{E}}$ mutant (Haitani et al., 2009). Interestingly, a novel mechanism, in which Rsp5 is dephosphorylated and activated when a rich nitrogen source is supplied and vice 
versa, was also proposed to involve the regulation of Gap1 ubiquitination (Sasaki and Takagi, 2013).

Moreover, the endocytosis of the maltose permease Mal61, the hexose transporter Hxt6/7, the uracil permease Fur4, the tryptophan permease Tat2, the zinc transporter Zrt1, and the divalent cation transporter $S m f 1$ is regulated via the ubiquitination by $\mathrm{Rsp} 5$ in response to various stimuli (Galan et al., 1996; Hein and Andre, 1997; Krampe et al., 1998; Medintz et al., 1998; Beck et al., 1999; Gitan and Eide, 2000; Sullivan et al., 2007; Nikko and Pelham, 2009). The internalization of the pheromone receptor Ste2 after pheromone sensing is also controlled by the ubiquitination mediated by Rsp5 as a part of its desensitization (Dunn and Hicke, 2001). In addition, Rsp5 ubiquitin ligase activity has been shown to be important for fluid phase endocytosis (Dunn and Hicke, 2001). Recently, the plasma membrane $\mathrm{H}^{+}$-ATPase Pmal was shown to be mono-ubiquitinated by Rsp5, leading to its internalization and vacuolar degradation, in response to the loss of V-ATPase activity (Smardon and Kane, 2014), suggesting that Rsp5 plays a role in the regulation of cytosolic $\mathrm{pH}$ homeostasis in yeast cells. In summary, Rsp5 plays important physiological roles in various cellular processes. However, our knowledge of the role of Rsp5 in regulation of the monovalent cation transporters Ena1/2/5 is still limited.

To catalyze the ubiquitination on a particular substrate, Rsp5 has to interact with the substrate through the interaction between the WW domains of Rsp5 and the PY motifs consisting of short peptide sequences (XPXY) located in the substrate. However,

TABLE 1 | Lists of Rsp5 adaptor proteins, conserved domains, and their locations.

\begin{tabular}{lll}
\hline Adaptor protein & Conserved domain & Location \\
\hline Bsd2 & TM & Golgi-endosome \\
Tre1/2 & TM, TFR dimer, PA & Golgi-endosome \\
Ear1/Ssh4 & TM, B30.2/SPRY & Golgi-endosome \\
Bul1/2 & Bul1 N-terminus, Bul1 & Plasma membrane, \\
& C-terminus & Golgi-endosome \\
Bul3 & Bul1 N-terminus & -ND- \\
Art1 & Arrestin N-terminus & Plasma membrane \\
Art2 & Arrestin C-terminus & Plasma membrane \\
Art3 & Arrestin N-terminus, & -ND- \\
& Arrestin C-terminus & \\
Art4 & Arrestin N-terminus, & Plasma membrane \\
& Arrestin C-terminus & \\
Art5 & Arrestin C-terminus & Plasma membrane \\
Art6 & Arrestin C-terminus & -ND- \\
Art7 & Arrestin N-terminus, & -ND- \\
& Arrestin C-terminus & \\
Art8 & Arrestin C-terminus & Plasma membrane \\
Art9 (Rim8) & Arrestin N-terminus, & Plasma membrane \\
Art10 & Arrestin C-terminus & \\
\hline & -ND- & -ND- \\
\hline
\end{tabular}

TM, Predicted transmembrane domain; TFR dimer, Transferrin receptor-like dimerization domain; PA, protease-associated domain; B30.2/SPRY, B30.2 and/or SP1a/RYanodine receptor domain; and ND, Not determined (Information was obtained from Saccharomyces Genome Database (SGD) and Lauwers et al., 2010). most of the Rsp5 substrates do not contain the PY motifs (Gupta et al., 2007), suggesting the requirement of PY-motifs containing adaptor proteins. The evidence supporting the existence of the Rsp5 adaptors originates from the identification of $\mathrm{Bsd} 2$ and Tre1/2, which contain the PY motifs, as the proteins required for the ubiquitination of the divalent cation transporter Smf1 (Liu et al., 1997; Hettema et al., 2004; Stimpson et al., 2006). In addition, Bsd2, but not Tre1/2, is found to be crucial for ubiquitination and trafficking of the precursor of the vacuolar enzyme polyphosphatase Phm5 (Hettema et al., 2004). This suggests that combination of adaptors can also affect the substrate specificity by Rsp5 (Watanabe et al., 2015). In addition, many Rsp5 adaptor proteins have been identified, including Bul1/2 (Soetens et al., 2001; Liu et al., 2007), Ear1 and its homologue Ssh4 (Leon et al., 2008), as well as the arrestin-related trafficking adaptor (ART) protein family members, which consist of Art1 to Art9 (Lin et al., 2008; Table 1).

\section{PROSPECTIVE ROLES OF Rsp5 AND ITS ADAPTORS IN SODIUM ACETATE RESPONSES}

We can summarize our understanding of the cellular responses to sodium acetate as follows. Previous studies have shown that acetate stress in the presence of sodium at $\mathrm{pH} 6.8$ exhibits a growth-inhibitory effect and triggers Hog1 MAPK

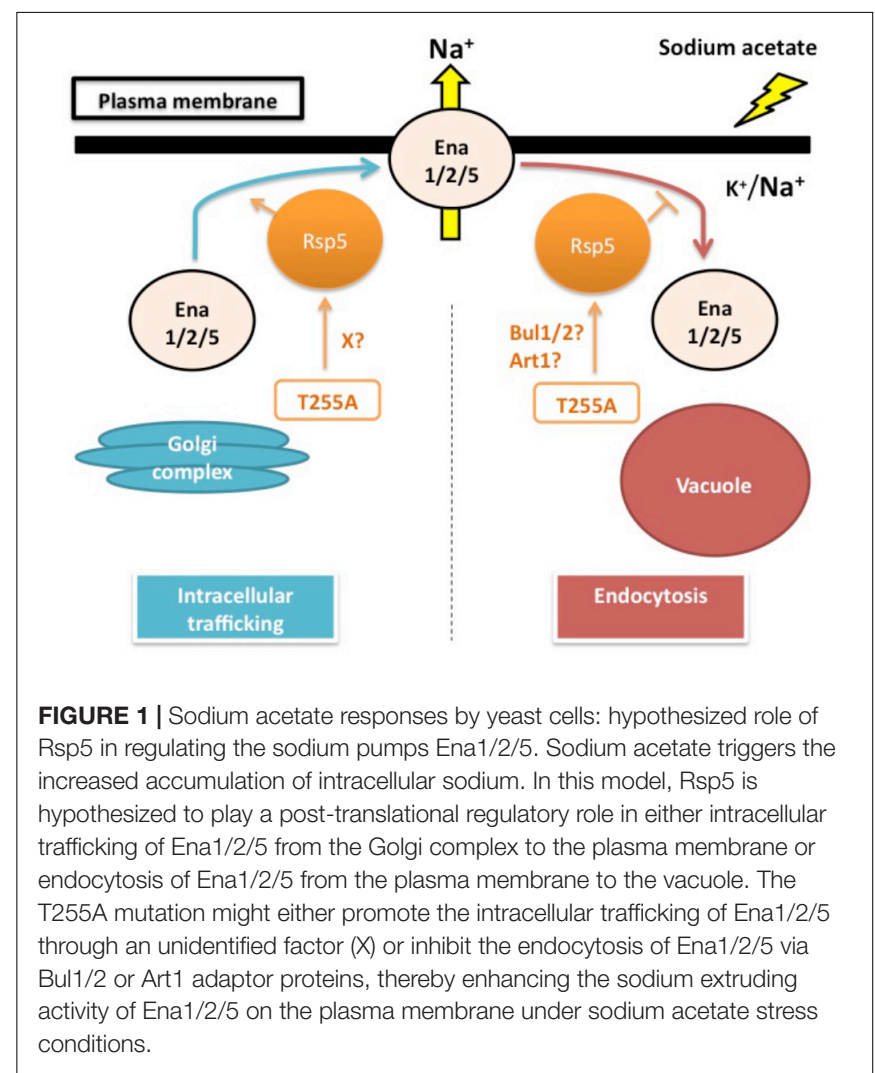


phosphorylation, which leads to upregulation of the GPD1 mRNA level and thereby increased accumulation of intracellular glycerol (Mollapour and Piper, 2006; Mollapour et al., 2009). Our study also found that disruption of HOG1 conferred sodium acetate sensitivity on yeast cells, but did not significantly affect the accumulation of intracellular sodium in yeast cells under sodium acetate stress conditions, suggesting that Hog1 mediated sodium acetate responses via other components e.g., glycerol (Watcharawipas et al., 2017). Thus, these studies indicate that one of the sodium acetate responses at higher $\mathrm{pH}$ is to increase intracellular osmolarity via the accumulation of glycerol, which allows the cells to counteract the loss of cytoplasmic water. Our recent study also revealed that the full acquisition of tolerance to sodium acetate is essentially dependent on the Rim101 pathway, since the disruption of RIM8, RIM20, and RIM101 causes sodium acetate sensitivity on yeast cells (Watcharawipas et al., 2017). Moreover, we found that yeast cells lacking RIM8 showed an increased accumulation of intracellular sodium content under sodium acetate stress conditions (Watcharawipas et al., 2017) similar to that observed under sodium chloride stress conditions (Marques et al., 2015), supporting the finding that the Rim101 pathway is crucial for the proper transport and accumulation of Enal on the plasma membrane (Marques et al., 2015). However, whether the Rim101 pathway has a principal role in regulating the transcription of ENA1 in the presence of sodium acetate still needs to be clarified. This might also explain why the disruption of HOG1 did not affect the intracellular sodium level under sodium acetate stress conditions. Thus, these results suggest that the HOG signaling and the Rim101 pathway independently play important roles in the sodium acetate responses in S. cerevisiae cells. Further investigation showed that the triple disruption of ENA1/2/5, which is downstream of the HOG and Rim101 pathways, confers sodium acetate sensitivity and increases the intracellular sodium accumulation in yeast cells under sodium acetate stress (Watcharawipas et al., 2017). Previous studies also show that the copy number of P-type ATPase sodium extrusion pump ENA1/2/5 genes is associated with acetate tolerance in the presence of sodium (Gilbert et al., 2009; Pena et al., 2013). Another study shows that the sodium/proton antiporters Nhal, Nhx1, and Vnx1 located at the plasma membrane, endosome, and vacuole, respectively, are involved in the cellular adaptation under sodium acetate stress at initial growth phase (Yang et al., 2010). Thus, these studies underscore the great importance of the Ena1/2/5 sodium pumps for the realization of sodium acetate tolerance, and imply that, at the very least, the sodium acetate responses involve the extrusion of sodium out of the cells or the sequestration of sodium inside the vacuole in the presence of acetate. Importantly, the intracellular sodium accumulation in the presence of sodium and acetate is higher than that in the presence of sodium and chloride, indicating the synergistic inhibitory effect of acetate anions (Watcharawipas et al., 2017). It would be intriguing to further investigate whether the acetate anions negatively impact the Ena1/2/5 activity.

The physiological importance of Rsp5 in the sodium acetate responses has been suggested by the sodium acetate sensitivity of $r s p 5^{\mathrm{L} 733 \mathrm{~S}}(r s p 5-1)$ and $r s p 5^{\mathrm{A} 401 \mathrm{E}}$, which have defects in the catalytic HECT domain and the WW3 domain, respectively (Watcharawipas et al., 2017). Recently, Wijayanti et al. (2015) found that Thr255Ala, an amino acid substitution in the WW1 domain, renders yeast cells the tolerance to sodium acetate with higher initial growth rate. Intriguingly, the changed threonine residue (Thr255) is conserved among the WW domains of Nedd4-family ubiquitin ligases and has corresponding positions at Thr357 in WW2 and Thr413 in WW3 of Rsp5. These threonine residues are also the putative phosphorylation sites which may play an important role in the substrate specificity of Rsp5 (Sasaki and Takagi, 2013; Watanabe et al., 2015). Interestingly, the Thr255Ala variant causes lower intracellular sodium accumulation than wild-type cells under sodium acetate stress. This sodium level difference is canceled by the triple deletion of ENA1/2/5, suggesting that the $r s p 5^{\mathrm{T} 255 \mathrm{~A}}$ mutant positively affects the sodium extruding activity through Ena1/2/5. Generally, Rsp5 post-translationally controls several plasma membrane proteins under various conditions. Thus, it is possible that Rsp5 might affect either Ena1/2/5 trafficking to the plasma membrane or Ena1/2/5 endocytosis. However, Ena1/2/5 does not have the PY motifs. For this reason, it is hypothesized that adaptor proteins are required for mediating the regulation of Ena1/2/5 by Rsp5. The Rsp 5 adaptors Rim8, Bul1/2 and Art1 have been shown to be important for the sodium acetate tolerance in yeast cells. However, the Rsp5-Rim8 interaction and Rim8 mono-ubiquitination by Rsp 5 are dispensable for sodium acetate tolerance (Watcharawipas et al., 2017). It would be intriguing to further examine the roles of Bul1/2 or Art1 in sodium acetate responses (Figure 1).

\section{CONCLUSION}

In this mini-review, we discuss the current understanding of sodium acetate stress as a composite stress of sodium and acetate, which may be able to influence each other. Thus, the cellular responses involve not only individual responses to sodium or to acetate, but also integrated actions to combat the effects of both. In addition, we shed light on a potentially important link-namely, that the protein ubiquitination system mediated by the E3 ubiquitin ligase Rsp5 possesses an important role in selectively regulating intracellular sodium homeostasis under sodium acetate stress, potentially through the P-type ATPases Ena1/2/5 and Rsp5 adaptor proteins. Further investigations will uncover the evolutionarily conserved role of Nedd4-family ubiquitin ligases, and will also benefit industrial applications through an improved understanding of their related stress conditions.

\section{AUTHOR CONTRIBUTIONS}

AW, DW, and HT analyzed the data and drafted the manuscript. AW prepared the figure and table. HT coordinated the manuscript preparation. All authors reviewed and approved the final version of manuscript. 


\section{REFERENCES}

Almeida, J. R. M., Modig, T., Petersson, A., Hahn-Hagerdal, B., Liden, G., and Gorwa-Grauslund, M. F. (2007). Increased tolerance and conversion of inhibitors in lignocellulosic hydrolysates by Saccharomyces cerevisiae. J. Chem. Technol. Biotechnol. 82, 340-349. doi: 10.1002/ jctb. 1676

Anwar, Z., Gulfraz, M., and Irshad, M. (2014). Agro-industrial lignocellulosic biomass a key to unlock the future bio-energy: a brief review. J. Radiat. Res. Appl. Sci. 7, 163-173. doi: 10.1016/j.jrras.2014.02.003

Arino, J., Ramos, J., and Sychrova, H. (2010). Alkali metal cation transport and homeostasis in yeasts. Microbiol. Mol. Biol. Rev. 74, 95-120. doi: 10.1128/ MMBR.00042-09

Arshad, M., Hussain, T., Iqbal, M., and Abbas, M. (2017). Enhanced ethanol production at commercial scale from molasses using high gravity technology by mutant S. cerevisiae. Braz. J. Microbiol. 48, 403-409. doi: 10.1016/j.bjm.2017. 02.003

Baek, S. H., Kwon, E. Y., Bae, S. J., Cho, B. R., Kim, S. Y., and Hahn, J. S. (2017). Improvement of D-lactic acid production in Saccharomyces cerevisiae under acidic conditions by evolutionary and rational metabolic engineering. Biotechnol. J. 12:1700015. doi: 10.1002/biot.201700015

Bazoti, S. F., Golunski, S., Pereira Siqueira, D., Scapini, T., Barrilli, E. T., Alex Mayer, D., et al. (2017). Second-generation ethanol from nondetoxified sugarcane hydrolysate by a rotting wood isolated yeast strain. Bioresour. Technol. 244, 582-587. doi: 10.1016/j.biortech.2017. 08.007

Beck, T., Schmidt, A., and Hall, M. N. (1999). Starvation induces vacuolar targeting and degradation of the tryptophan permease in yeast. J. Cell Biol. 146, 12271237. doi: $10.1083 /$ jcb.146.6.1227

Bellissimi, E., van Dijken, J. P., Pronk, J. T., and van Maris, A. J. (2009). Effects of acetic acid on the kinetics of xylose fermentation by an engineered, xyloseisomerase-based Saccharomyces cerevisiae strain. FEMS Yeast Res. 9, 358-364. doi: 10.1111/j.1567-1364.2009.00487.x

Cagnac, O., Leterrier, M., Yeager, M., and Blumwald, E. (2007). Identification and characterization of Vnxlp, a novel type of vacuolar monovalent cation/H + antiporter of Saccharomyces cerevisiae. J. Biol. Chem. 282, 24284-24293. doi: 10.1074/jbc.M703116200

Dechant, R., Binda, M., Lee, S. S., Pelet, S., Winderickx, J., and Peter, M. (2010). Cytosolic $\mathrm{pH}$ is a second messenger for glucose and regulates the PKA pathway through V-ATPase. EMBO J. 29, 2515-2526. doi: 10.1038/emboj. 2010.138

Dihazi, H., Kessler, R., and Eschrich, K. (2004). High osmolarity glycerol (HOG) pathway-induced phosphorylation and activation of 6-phosphofructo2-kinase are essential for glycerol accumulation and yeast cell proliferation under hyperosmotic stress. J. Biol. Chem. 279, 23961-23968. doi: 10.1074/jbc. M312974200

Ding, J., Bierma, J., Smith, M. R., Poliner, E., Wolfe, C., Hadduck, A. N., et al. (2013). Acetic acid inhibits nutrient uptake in Saccharomyces cerevisiae: auxotrophy confounds the use of yeast deletion libraries for strain improvement. Appl. Microbiol. Biotechnol. 97, 7405-7416. doi: 10.1007/s00253013-5071-y

Dunn, R., and Hicke, L. (2001). Domains of the Rsp5 ubiquitin-protein ligase required for receptor-mediated and fluid-phase endocytosis. Mol. Biol. Cell 12, 421-435. doi: 10.1091/mbc.12.2.421

Fernandes, A. R., Mira, N. P., Vargas, R. C., Canelhas, I., and Sa-Correia, I. (2005). Saccharomyces cerevisiae adaptation to weak acids involves the transcription factor Haalp and Haalp-regulated genes. Biochem. Biophys. Res. Commun. 337, 95-103. doi: 10.1016/j.bbrc.2005.09.010

Fernandez-Nino, M., Marquina, M., Swinnen, S., Rodriguez-Porrata, B., Nevoigt, E., and Arino, J. (2015). The cytosolic pH of individual Saccharomyces cerevisiae cells is a key factor in acetic acid tolerance. Appl. Environ. Microbiol. 81, 7813-7821. doi: 10.1128/AEM.02313-15

Ferreira, C., van Voorst, F., Martins, A., Neves, L., Oliveira, R., Kielland-Brandt, M. C., et al. (2005). A member of the sugar transporter family, Stllp is the glycerol/H + symporter in Saccharomyces cerevisiae. Mol. Biol. Cell. 16, 2068-2076. doi: 10.1091/mbc.e04-10-0884

Galan, J. M., Moreau, V., Andre, B., Volland, C., and Haguenauer-Tsapis, R. (1996). Ubiquitination mediated by the Npilp/Rsp5p ubiquitin-protein ligase is required for endocytosis of the yeast uracil permease. J. Biol. Chem. 271, 10946-10952. doi: 10.1074/jbc.271.18.10946

Generoso, W. C., Brinek, M., Dietz, H., Oreb, M., and Boles, E. (2017). Secretion of 2,3-dihydroxyisovalerate as a limiting factor for isobutanol production in Saccharomyces cerevisiae. FEMS Yeast Res. 17:fox029. doi: 10.1093/femsyr/ fox029

Gilbert, A., Sangurdekar, D. P., and Srienc, F. (2009). Rapid strain improvement through optimized evolution in the cytostat. Biotechnol. Bioeng. 103, 500-512. doi: 10.1002/bit.22272

Gitan, R. S., and Eide, D. J. (2000). Zinc-regulated ubiquitin conjugation signals endocytosis of the yeast ZRT1 zinc transporter. Biochem. J. 346, 329-336. doi: 10.1042/bj3460329

Gomez, M. J., Luyten, K., and Ramos, J. (1996). The capacity to transport potassium influences sodium tolerance in Saccharomyces cerevisiae. FEMS Microbiol. Lett. 135, 157-160. doi: 10.1016/0378-1097(95) 00441-6

Guo, Z., and Olsson, L. (2014). Physiological response of Saccharomyces cerevisiae to weak acids present in lignocellulosic hydrolysate. FEMS Yeast Res. 14, 1234-1248. doi: 10.1111/1567-1364.12221

Gupta, R., Kus, B., Fladd, C., Wasmuth, J., Tonikian, R., Sidhu, S., et al. (2007). Ubiquitination screen using protein microarrays for comprehensive identification of Rsp5 substrates in yeast. Mol. Syst. Biol. 3:116. doi: 10.1038/ msb4100159

Haitani, Y., Nakata, M., Sasaki, T., Uchida, A., and Takagi, H. (2009). Engineering of the yeast ubiquitin ligase Rsp5: isolation of a new variant that induces constitutive inactivation of the general amino acid permease Gap1. FEMS Yeast Res. 9, 73-86. doi: 10.1111/j.1567-1364.2008.00460.x

Haitani, Y., Shimoi, H., and Takagi, H. (2006). Rsp5 regulates expression of stress proteins via post-translational modification of Hsf1 and Msn4 in Saccharomyces cerevisiae. FEBS Lett. 580, 3433-3438. doi: 10.1016/j.febslet.2006.05.016

Haitani, Y., and Takagi, H. (2008). Rsp5 is required for the nuclear export of mRNA of HSF1 and MSN2/4 under stress conditions in Saccharomyces cerevisiae. Genes Cells 13, 105-116. doi: 10.1111/j.1365-2443.2007.01154.x

Haro, R., and Rodriguez-Navarro, A. (2002). Molecular analysis of the mechanism of potassium uptake through the TRK1 transporter of Saccharomyces cerevisiae. Biochim. Biophys. Acta 1564, 114-122. doi: 10.1016/S0005-2736(02) 00408-X

Hein, C., and Andre, B. (1997). A C-terminal di-leucine motif and nearby sequences are required for $\mathrm{NH}_{4}^{+}$-induced inactivation and degradation of the general amino acid permease, Gaplp, of Saccharomyces cerevisiae. Mol. Microbiol. 24, 607-616. doi: 10.1046/j.1365-2958.1997.3771735.x

Hettema, E. H., Valdez-Taubas, J., and Pelham, H. R. (2004). Bsd2 binds the ubiquitin ligase Rsp5 and mediates the ubiquitination of transmembrane proteins. EMBO J. 23, 1279-1288. doi: 10.1038/sj.emboj. 7600137

Hiraishi, H., Shimada, T., Ohtsu, I., Sato, T., and Takagi, H. (2009). The yeast ubiquitin ligase Rsp5 down-regulates the alpha subunit of nascent polypeptideassociated complex Egd2 under stress conditions. FEBS J. 276, 5287-5297. doi: $10.1111 / \mathrm{j} .1742-4658.2009 .07226 . \mathrm{x}$

Hohmann, S. (2002). Osmotic stress signaling and osmoadaptation in yeasts. Microbiol. Mol. Biol. Rev. 66, 300-372. doi: 10.1128/mmbr.66.2.300372.2002

Hoppe, T., Matuschewski, K., Rape, M., Schlenker, S., Ulrich, H. D., and Jentsch, S. (2000). Activation of a membrane-bound transcription factor by regulated ubiquitin/proteasome-dependent processing. Cell 102, 577-586. doi: 10.1016/ S0092-8674(00)00080-5

Hoshikawa, C., Shichiri, M., Nakamori, S., and Takagi, H. (2003). A nonconserved Ala401 in the yeast Rsp5 ubiquitin ligase is involved in degradation of Gap1 permease and stress-induced abnormal proteins. Proc. Natl. Acad. Sci. U.S.A. 100, 11505-11510. doi: 10.1073/pnas.1933 153100

Inaba, T., Watanabe, D., Yoshiyama, Y., Tanaka, K., Ogawa, J., Takagi, H., et al. (2013). An organic acid-tolerant HAA1-overexpression mutant of an industrial bioethanol strain of Saccharomyces cerevisiae and its application to the production of bioethanol from sugarcane molasses. AMB Express 3, 1-7. doi: 10.1186/2191-0855-3-74

Ishida, Y., Nguyen, T. T. M., and Izawa, S. (2017). The yeast ADH7 promoter enables gene expression under pronounced translation 
repression caused by the combined stress of vanillin, furfural, and 5hydroxymethylfurfural. J. Biotechnol. 252, 65-72. doi: 10.1016/j.jbiotec.2017. 04.024

Iwaki, A., Ohnuki, S., Suga, Y., Izawa, S., and Ohya, Y. (2013). Vanillin inhibits translation and induces messenger ribonucleoprotein (mRNP) granule formation in Saccharomyces cerevisiae: application and validation of highcontent, image-based profiling. PLoS One 8:e61748. doi: 10.1371/journal.pone. 0061748

Jarmoszewicz, K., Lukasiak, K., Riezman, H., and Kaminska, J. (2012). Rsp5 ubiquitin ligase is required for protein trafficking in Saccharomyces cerevisiae COPI mutants. PLoS One 7:e39582. doi: 10.1371/journal.pone. 0039582

Jayakody, L. N., Ferdouse, J., Hayashi, N., and Kitagaki, H. (2017). Identification and detoxification of glycolaldehyde, an unattended bioethanol fermentation inhibitor. Crit. Rev. Biotechnol. 37, 177-189. doi: 10.3109/07388551.2015. 1128877

Jayakody, L. N., Turner, T. L., Yun, E. J., Kong, I. I., Liu, J. J., and Jin, Y. S. (2018). Expression of Gre2p improves tolerance of engineered xylose-fermenting Saccharomyces cerevisiae to glycolaldehyde under xylose metabolism. Appl. Microbiol. Biotechnol. 102, 8121-8133. doi: 10.1007/s00253-018-9216-x

Jonsson, L. J., and Martin, C. (2016). Pretreatment of lignocellulose: formation of inhibitory by-products and strategies for minimizing their effects. Bioresour. Technol. 199, 103-112. doi: 10.1016/j.biortech.2015.10.009

Kawahata, M., Masaki, K., Fujii, T., and Iefuji, H. (2006). Yeast genes involved in response to lactic acid and acetic acid: acidic conditions caused by the organic acids in Saccharomyces cerevisiae cultures induce expression of intracellular metal metabolism genes regulated by Aft1p. FEMS Yeast Res. 6, 924-936. doi: 10.1111/j.1567-1364.2006.00089.x

Kitichantaropas, Y., Boonchird, C., Sugiyama, M., Kaneko, Y., Harashima, S., and Auesukaree, C. (2016). Cellular mechanisms contributing to multiple stress tolerance in Saccharomyces cerevisiae strains with potential use in hightemperature ethanol fermentation. AMB Express 6:107. doi: 10.1186/s13568016-0285-x

Klinke, H. B., Thomsen, A. B., and Ahring, B. K. (2004). Inhibition of ethanolproducing yeast and bacteria by degradation products produced during pretreatment of biomass. Appl. Microbiol. Biotechnol. 66, 10-26. doi: 10.1007/ s00253-004-1642-2

Krampe, S., Stamm, O., Hollenberg, C. P., and Boles, E. (1998). Catabolite inactivation of the high-affinity hexose transporters Hxt6 and Hxt7 of Saccharomyces cerevisiae occurs in the vacuole after internalization by endocytosis. FEBS Lett. 441, 343-347. doi: 10.1016/S0014-5793(98) 01583-X

Lamb, T. M., Xu, W., Diamond, A., and Mitchell, A. P. (2001). Alkaline response genes of Saccharomyces cerevisiae and their relationship to the RIM101 pathway. J. Biol. Chem. 276, 1850-1856. doi: 10.1074/jbc.M008381200

Lapathitis, G., and Kotyk, A. (1998). Univalent cation fluxes in yeast. Biochem. Mol. Biol. Int. 44, 371-380.

Larsson, S., Palmqvist, E., Hahn-Hagerdal, B., Tengborg, C., Stenberg, K., Zacchi, G., et al. (1999). The generation of fermentation inhibitors during dilute acid hydrolysis of softwood. Enzyme Microb. Technol. 24, 151-159. doi: 10.1016/S0141-0229(98)00101-X

Lauwers, E., Erpapazoglou, Z., Haguenauer-Tsapis, R., and Andre, B. (2010). The ubiquitin code of yeast permease trafficking. Trends Cell Biol. 20, 196-204. doi: $10.1016 /$ j.tcb.2010.01.004

Lee, J., Reiter, W., Dohnal, I., Gregori, C., Beese-Sims, S., Kuchler, K., et al. (2013). MAPK Hog1 closes the S. cerevisiae glycerol channel Fps1 by phosphorylating and displacing its positive regulators. Genes Dev. 27, 2590-2601. doi: 10.1101/ gad.229310.113

Leon, S., Erpapazoglou, Z., and Haguenauer-Tsapis, R. (2008). Ear1p and Ssh4p are new adaptors of the ubiquitin ligase Rsp5p for cargo ubiquitylation and sorting at multivesicular bodies. Mol. Biol. Cell 19, 2379-2388. doi: 10.1091/mbc.E0801-0068

Limayem, A., and Ricke, S. C. (2012). Lignocellulosic biomass for bioethanol production: current perspectives, potential issues and future prospects. Prog. Energy Combust. Sci. 38, 449-467. doi: 10.1016/j.pecs.2012.03.002

Lin, C. H., MacGurn, J. A., Chu, T., Stefan, C. J., and Emr, S. D. (2008). Arrestinrelated ubiquitin-ligase adaptors regulate endocytosis and protein turnover at the cell surface. Cell 135, 714-725. doi: 10.1016/j.cell.2008.09.025
Liu, J., Sitaram, A., and Burd, C. G. (2007). Regulation of copper-dependent endocytosis and vacuolar degradation of the yeast copper transporter, Ctr1p, by the Rsp5 ubiquitin ligase. Traffic 8, 1375-1384. doi: 10.1111/j.1600-0854.2007. 00616.x

Liu, X. F., Supek, F., Nelson, N., and Culotta, V. C. (1997). Negative control of heavy metal uptake by the Saccharomyces cerevisiae BSD2 gene. J. Biol. Chem. 272, 11763-11769. doi: 10.1074/jbc.272.18.11763

Ludovico, P., Rodrigues, F., Almeida, A., Silva, M. T., Barrientos, A., and CorteReal, M. (2002). Cytochrome c release and mitochondria involvement in programmed cell death induced by acetic acid in Saccharomyces cerevisiae. Mol. Biol. Cell 13, 2598-2606. doi: 10.1091/mbc.E01-12-0161

Marques, M. C., Zamarbide-Fores, S., Pedelini, L., Llopis-Torregrosa, V., and Yenush, L. (2015). A functional Rim101 complex is required for proper accumulation of the Enal $\mathrm{Na}^{+}$-ATPase protein in response to salt stress in Saccharomyces cerevisiae. FEMS Yeast Res. 15:fov017. doi: 10.1093/femsyr/ fov017

Martinez, P., and Persson, B. L. (1998). Identification, cloning and characterization of a derepressible $\mathrm{Na}^{+}$-coupled phosphate transporter in Saccharomyces cerevisiae. Mol. Gen. Genet. 258, 628-638. doi: 10.1007/s0043800 50776

Martinez-Munoz, G. A., and Kane, P. (2008). Vacuolar and plasma membrane proton pumps collaborate to achieve cytosolic $\mathrm{pH}$ homeostasis in yeast. J. Biol. Chem. 283, 20309-20319. doi: 10.1074/jbc.M710470200

Medintz, I., Jiang, H., and Michels, C. A. (1998). The role of ubiquitin conjugation in glucose-induced proteolysis of Saccharomyces maltose permease. J. Biol. Chem. 273, 34454-34462. doi: 10.1074/jbc.273.51.34454

Mira, N. P., Becker, J. D., and Sa-Correia, I. (2010). Genomic expression program involving the Haalp-regulon in Saccharomyces cerevisiae response to acetic acid. OMICS 14, 587-601. doi: 10.1089/omi.2010.0048

Mira, N. P., Henriques, S. F., Keller, G., Teixeira, M. C., Matos, R. G., Arraiano, C. M., et al. (2011). Identification of a DNA-binding site for the transcription factor Haal, required for Saccharomyces cerevisiae response to acetic acid stress. Nucleic Acids Res. 39, 6896-6907. doi: 10.1093/nar/ gkr228

Mira, N. P., Lourenco, A. B., Fernandes, A. R., Becker, J. D., and Sa-Correia, I. (2009). The RIM101 pathway has a role in Saccharomyces cerevisiae adaptive response and resistance to propionic acid and other weak acids. FEMS Yeast Res. 9, 202-216. doi: 10.1111/j.1567-1364.2008.00473.x

Mollapour, M., and Piper, P. W. (2006). Hog1p mitogen-activated protein kinase determines acetic acid resistance in Saccharomyces cerevisiae. FEMS Yeast Res. 6, 1274-1280. doi: 10.1111/j.1567-1364.2006.00118.x

Mollapour, M., and Piper, P. W. (2007). Hog1 mitogen-activated protein kinase phosphorylation targets the yeast Fps1 aquaglyceroporin for endocytosis, thereby rendering cells resistant to acetic acid. Mol. Cell. Biol. 27, 6446-6456. doi: 10.1128/MCB.02205-06

Mollapour, M., Shepherd, A., and Piper, P. W. (2008). Novel stress responses facilitate Saccharomyces cerevisiae growth in the presence of the monocarboxylate preservatives. Yeast 25, 169-177. doi: 10.1002/yea.1576

Mollapour, M., Shepherd, A., and Piper, P. W. (2009). Presence of the Fps1p aquaglyceroporin channel is essential for Hoglp activation, but suppresses Slt2(Mpk1)p activation, with acetic acid stress of yeast. Microbiology 155, 3304-3311. doi: 10.1099/mic.0.030502-0

Mongkontanawat, N., Wasikadilok, N., Phuangborisut, S., Chanawanno, T., and Khunphutthiraphi, T. (2018). B-Glucan production of Saccharomyces cerevisiae by using malva nut juice production wastewater. Int. Food Res. J. 25, 499-503.

Murguia, J. R., Belles, J. M., and Serrano, R. (1996). The yeast HAL2 nucleotidase is an in vivo target of salt toxicity. J. Biol. Chem. 271, 29029-29033. doi: 10.1074/ jbc.271.46.29029

Nakagawa, Y., Seita, J., Komiyama, S., Yamamura, H., Hayakawa, M., and Iimura, Y. (2013). A new simple method for isolating multistress-tolerant semidominant mutants of Saccharomyces cerevisiae by one-step selection under lethal hydrogen peroxide stress condition. Biosci. Biotechnol. Biochem. 77, 224-228. doi: 10.1271/bbb.120533

Nass, R., Cunningham, K. W., and Rao, R. (1997). Intracellular sequestration of sodium by a novel $\mathrm{Na}^{+} / \mathrm{H}^{+}$exchanger in yeast is enhanced by mutations in the plasma membrane $\mathrm{H}^{+}$-ATPase. J. Biol. Chem. 272, 26145-26152. doi: 10.1074/ jbc.272.42.26145 
Nikko, E., and Pelham, H. R. (2009). Arrestin-mediated endocytosis of yeast plasma membrane transporters. Traffic 10, 1856-1867. doi: 10.1111/j.1600-0854.2009. 00990.x

Orij, R., Urbanus, M. L., Vizeacoumar, F. J., Giaever, G., Boone, C., Nislow, C., et al. (2012). Genome-wide analysis of intracellular $\mathrm{pH}$ reveals quantitative control of cell division rate by pHc in Saccharomyces cerevisiae. Genome Biol. 13, 1-15. doi: $10.1186 / \mathrm{gb}-2012-13-9-\mathrm{r} 80$

Palmqvist, E., and Hahn-Hagerdal, B. (2000). Fermentation of lignocellulosic hydrolysates. II: inhibitors and mechanisms of inhibition. Bioresour. Technol. 74, 25-33. doi: 10.1016/S0960-8524(99)00161-3

Pampulha, M. E., and Loureiro-Dias, M. C. (1989). Combined effect of acetic acid, $\mathrm{pH}$ and ethanol on intracellular $\mathrm{pH}$ of fermenting yeast. Appl. Microbiol. Biotechnol. 31, 547-550. doi: 10.1007/BF00270792

Pena, P. V., Glasker, S., and Srienc, F. (2013). Genome-wide overexpression screen for sodium acetate resistance in Saccharomyces cerevisiae. J. Biotechnol. 164, 26-33. doi: 10.1016/j.jbiotec.2012.12.005

Petelenz-Kurdziel, E., Kuehn, C., Nordlander, B., Klein, D., Hong, K. K., Jacobson, T., et al. (2013). Quantitative analysis of glycerol accumulation, glycolysis and growth under hyper osmotic stress. PLoS Comput. Biol. 9:e1003084. doi: 10.1371/journal.pcbi.1003084

Proft, M., and Serrano, R. (1999). Repressors and upstream repressing sequences of the stress regulated ENA1 gene in Saccharomyces cerevisiae: bZIP protein Skolp confers HOG-dependent osmotic regulation. Mol. Cell. Biol. 19, 537-546. doi: 10.1128/MCB.19.1.537

Proft, M., and Struhl, K. (2002). Hog1 kinase converts the Sko1-Cyc8-Tup1 repressor complex into an activator that recruits SAGA and SWI/SNF in response to osmotic stress. Mol. Cell 9, 1307-1317. doi: 10.1016/S1097-2765(02) 00557-9

Proft, M., and Struhl, K. (2004). MAP kinase-mediated stress relief that precedes and regulates the timing of transcriptional induction. Cell 118, 351-361. doi: 10.1016/j.cell.2004.07.016

Rep, M., Krantz, M., Thevelein, J. M., and Hohmann, S. (2000). The transcriptional response of Saccharomyces cerevisiae to osmotic shock. J. Biol. Chem. 275, 8290-8300. doi: 10.1074/jbc.275.12.8290

Rotin, D., and Kumar, S. (2009). Physiological functions of the HECT family of ubiquitin ligases. Nat. Rev. Mol. Cell Biol. 10, 398-409. doi: 10.1038/ nrm 2690

Sasaki, T., and Takagi, H. (2013). Phosphorylation of a conserved Thr357 in yeast Nedd4-like ubiquitin ligase Rsp5 is involved in downregulation of the general amino acid permease Gap1. Genes Cells 18, 459-475. doi: 10.1111/gtc. 12049

Sasano, Y., Haitani, Y., Hashida, K., Ohtsu, I., Shima, J., and Takagi, H. (2012a). Enhanement of the proline and nitric oxide synthetic pathway improves fermentation ability under multiple baking-associated stress conditions in industrial baker's yeast. Microb. Cell Fact. 11:40. doi: 10.1186/1475-2859-11-40

Sasano, Y., Watanabe, D., Ukibe, K., Inai, T., Ohtsu, I., Shimoi, H., et al. (2012b). Overexpression of the yeast transcription activator Msn2 confers furfural resistance and increases the initial fermentation rate in ethanol production. J. Biosci. Bioeng. 113, 451-455. doi: 10.1016/j.jbiosc.2011.11.017

Serrano, R., Ruiz, A., Bernal, D., Chambers, J. R., and Arino, J. (2002). The transcriptional response to alkaline $\mathrm{pH}$ in Saccharomyces cerevisiae: evidence for calcium-mediated signalling. Mol. Microbiol. 46, 1319-1333. doi: 10.1046/j. 1365-2958.2002.03246.x

Shcherbik, N., Kee, Y., Lyon, N., Huibregtse, J. M., and Haines, D. S. (2004). A single PXY motif located within the carboxyl terminus of Spt23p and Mga2p mediates a physical and functional interaction with ubiquitin ligase Rsp5p. J. Biol. Chem. 279, 53892-53898. doi: 10.1074/jbc.M410325200

Shcherbik, N., Zoladek, T., Nickels, J. T., and Haines, D. S. (2003). Rsp5p is required for ER bound Mga2p120 polyubiquitination and release of the processed/tethered transactivator Mga2p90. Curr. Biol. 13, 1227-1233. doi: 10. 1016/s0960-9822(03)00457-3

Shiga, T., Yoshida, N., Shimizu, Y., Suzuki, E., Sasaki, T., Watanabe, D., et al. (2014). Quality control of plasma membrane proteins by Saccharomyces cerevisiae Nedd4-like ubiquitin ligase Rsp5p under environmental stress conditions. Eukaryot. Cell 13, 1191-1199. doi: 10.1128/EC.00104-14

Shima, J., and Takagi, H. (2009). Stress-tolerance of baker's-yeast (Saccharomyces cerevisiae) cells: stress-protective molecules and genes involved in stress tolerance. Biotechnol. Appl. Biochem. 53, 155-164. doi: 10.1042/BA20090029
Shiroma, S., Jayakody, L. N., Horie, K., Okamoto, K., and Kitagaki, H. (2014). Enhancement of ethanol fermentation in Saccharomyces cerevisiae sake yeast by disrupting mitophagy function. Appl. Environ. Microbiol. 80, 1002-1012. doi: 10.1128/AEM.03130-13

Simoes, T., Mira, N. P., Fernandes, A. R., and Sa-Correia, I. (2006). The SPI1 gene, encoding a glycosylphosphatidylinositol-anchored cell wall protein, plays a prominent role in the development of yeast resistance to lipophilic weak-acid food preservatives. Appl. Environ. Microbiol. 72, 7168-7175. doi: 10.1128/AEM. 01476-06

Smardon, A. M., and Kane, P. M. (2014). Loss of vacuolar $\mathrm{H}^{+}$-ATPase activity in organelles signals ubiquitination and endocytosis of the yeast plasma membrane proton pump Pmalp. J. Biol. Chem. 289, 32316-32326. doi: 10.1074/jbc.M114. 574442

Soetens, O., De Craene, J. O., and Andre, B. (2001). Ubiquitin is required for sorting to the vacuole of the yeast general amino acid permease, Gap1. J. Biol. Chem. 276, 43949-43957. doi: 10.1074/jbc.M102945200

Springael, J. Y., Galan, J. M., Haguenauer-Tsapis, R., and Andre, B. (1999). NH4 ${ }^{+}$induced down-regulation of the Saccharomyces cerevisiae Gaplp permease involves its ubiquitination with lysine-63-linked chains. J. Cell Sci. 112, 13751383.

Stimpson, H. E., Lewis, M. J., and Pelham, H. R. (2006). Transferrin receptorlike proteins control the degradation of a yeast metal transporter. EMBO J. 25, 662-672. doi: 10.1038/sj.emboj.7600984

Stratford, M., Nebe-von-Caron, G., Steels, H., Novodvorska, M., Ueckert, J., and Archer, D. B. (2013). Weak-acid preservatives: $\mathrm{pH}$ and proton movements in the yeast Saccharomyces cerevisiae. Int. J. Food Microbiol. 161, 164-171. doi: 10.1016/j.ijfoodmicro.2012.12.013

Sugiyama, M., Akase, S. P., Nakanishi, R., Horie, H., Kaneko, Y., and Harashima, S. (2014). Nuclear localization of Haal, which is linked to its phosphorylation status, mediates lactic acid tolerance in Saccharomyces cerevisiae. Appl. Environ. Microbiol. 80, 3488-3495. doi: 10.1128/AEM. 04241-13

Sullivan, J. A., Lewis, M. J., Nikko, E., and Pelham, H. R. B. (2007). Multiple interactions drive adaptor-mediated recruitment of the ubiquitin ligase Rsp5 to membrane proteins in vivo and in vitro. Mol. Biol. Cell 18, 2429-2440. doi: 10.1091/mbc.E07-01-0011

Takpho, N., Watanabe, D., and Takagi, H. (2018). High-level production of valine by expression of the feedback inhibition-insensitive acetohydroxyacid synthase in Saccharomyces cerevisiae. Metab. Eng. 46, 60-67. doi: 10.1016/j.ymben.2018. 02.011

Tanaka, K., Ishii, Y., Ogawa, J., and Shima, J. (2012). Enhancement of acetic acid tolerance in Saccharomyces cerevisiae by overexpression of the HAAl gene, encoding a transcriptional activator. Appl. Environ. Microbiol. 78, 8161-8163. doi: 10.1128/AEM.02356-12

Ullah, A., Chandrasekaran, G., Brul, S., and Smits, G. J. (2013). Yeast adaptation to weak acids prevents futile energy expenditure. Front. Microbiol. 4:142. doi: $10.3389 /$ fmicb.2013.00142

Ullah, A., Orij, R., Brul, S., and Smits, G. J. (2012). Quantitative analysis of the modes of growth inhibition by weak organic acids in Saccharomyces cerevisiae. Appl. Environ. Microbiol. 78, 8377-8387. doi: 10.1128/AEM. 02126-12

Watanabe, D., Murai, H., Tanahashi, R., Nakamura, K., Sasaki, T., and Takagi, H. (2015). Cooperative and selective roles of the WW domains of the yeast Nedd4like ubiquitin ligase Rsp5 in the recognition of the arrestin-like adaptors Bul1 and Bul2. Biochem. Biophys. Res. Commun. 463, 76-81. doi: 10.1016/j.bbrc.2015. 05.025

Watcharawipas, A., Watanabe, D., and Takagi, H. (2017). Enhanced sodium acetate tolerance in Saccharomyces cerevisiae by the Thr255Ala mutation of the ubiquitin ligase Rsp5. FEMS Yeast Res. 17:fox083. doi: 10.1093/femsyr/ fox 083

Wijayanti, I., Watanabe, D., Oshiro, S., and Takagi, H. (2015). Isolation and functional analysis of yeast ubiquitin ligase Rsp5 variants that alleviate the toxicity of human $\alpha$-synuclein. J. Biochem. 157, 251-260. doi: 10.1093/jb/ mvu069

Wilkinson, S., Greetham, D., and Tucker, G. A. (2016). Evaluation of different lignocellulosic biomass pretreatments by phenotypic microarraybased metabolic analysis of fermenting yeast. Biofuel Res. J. 3, 357-365. doi: 10.18331/brj2016.3.1.5 
Yang, S., Land, M. L., Klingeman, D. M., Pelletier, D. A., Lu, T.-Y. S., Martin, S. L., et al. (2010). Paradigm for industrial strain improvement identifies sodium acetate tolerance loci in Zymomonas mobilis and Saccharomyces cerevisiae. Proc. Natl. Acad. Sci. U.S.A. 107, 10395-10400. doi: 10.1073/pnas.0914506107

Young, B. P., Shin, J. J. H., Orij, R., Chao, J. T., Li, S. C., Guan, X. L., et al. (2010). Phosphatidic acid is a $\mathrm{pH}$ biosensor that links membrane biogenesis to metabolism. Science 329, 1085-1088. doi: 10.1126/science.1191026

Zhang, S., Skalsky, Y., and Garfinkel, D. J. (1999). MGA2 or SPT23 is required for transcription of the D9 fatty acid desaturase gene, OLE1, and nuclear membrane integrity in Saccharomyces cerevisiae. Genetics 151, $473-483$.
Conflict of Interest Statement: The authors declare that the research was conducted in the absence of any commercial or financial relationships that could be construed as a potential conflict of interest.

Copyright (c) 2018 Watcharawipas, Watanabe and Takagi. This is an open-access article distributed under the terms of the Creative Commons Attribution License (CC BY). The use, distribution or reproduction in other forums is permitted, provided the original author(s) and the copyright owner(s) are credited and that the original publication in this journal is cited, in accordance with accepted academic practice. No use, distribution or reproduction is permitted which does not comply with these terms. 